\title{
Simple and Rapid Determination of Diuretics by Luminescent Method
}

\author{
Iuna Tsyrulneva, Olga Zaporozhets
}

Chemistry Department, Taras Shevchenko National University of Kyiv, Kyiv, Ukraine.

Email: iuna_tsyrulneva@yahoo.com

Received August $2^{\text {nd }}, 2013$; revised August $6^{\text {th }}, 2013$; accepted September $18^{\text {th }}, 2013$

Copyright (C) 2013 Iuna Tsyrulneva, Olga Zaporozhets. This is an open access article distributed under the Creative Commons Attribution License, which permits unrestricted use, distribution, and reproduction in any medium, provided the original work is properly cited.

\begin{abstract}
Diuretics are drugs widely used in treatment of heart failure and hypertension and as doping agents in sports. Wrong prescription and excessive abuse can lead to negative side effects. Despite the effectiveness of methods usually used for the determination of diuretics (gas or liquid chromatography, capillary electrophoresis), they do not always provide necessary sensitivity. Moreover, sample preparation increases time of analysis. A rapid and sensitive luminescent method for determination of 5 diuretics (amiloride, bendroflumethiazide, bumetanide, furosemide, triamterene) in aqueous solutions and amiloride and triamterene in human urine is described. Intrinsic luminescent properties of protolytic forms of diuretics were studied in order to provide highly sensitive analysis. Investigation of interfering influence of diuretics was carried out to provide selective determination of triamterene, bumetanide and furosemide in aqueous mixtures of diuretics. Influence of urine at luminescent properties of diuretics was studied. The possibility of determination of triamterene and amiloride in human urine as individual substances and in mixture was proved. Simple and rapid technique for their determination in human urine was elaborated. The techniques elaborated for determination of triamterene in presence of other diuretics and furosemide in presence of commensurate amount of bumetanide allow enhancing specifity of analysis. Sufficient selectivity and sensitivity were reached in determination of amiloride and triamterene in human urine. The reduction of time of analysis due to avoiding sample preparation merits the techniques proposed.
\end{abstract}

Keywords: Furosemide; Bumetanide; Amiloride; Triamterene; Thiazides; Separation; Luminescence; Human Urine

\section{Introduction}

Diuretics are drugs that increase the amount of urine produced and enhance the excretion of electrolytes and water with urine as a result of disruption of ion transport in the kidney [1]. They are widely used in clinical practice in the treatment of hypertension and in different kinds of edema as well as for the correction of acid-base balance, in the treatment of intoxication and some injuries. Despite their effectiveness, diuretics may cause serious problems in case of wrong administration and excessive abuse [2]. Thus, it is of primary importance to control the intake of diuretics in order to avoid negative side effects.

Diuretics are prohibited for samples taken out of and in competition according to World Anti-Doping Agency (WADA) [3]. In sports, athletes misuse diuretics for several reasons: to reduce body weight in order to qualify for a lower weight, to reduce the urinary concentration of other prohibited substances to avoid a positive doping result and to overcome fluid retention caused by the use of anabolic steroids. Diuretics need to be detected at or a lower level than the minimum required performance limit (MRPL) $-250 \mathrm{ng} \cdot \mathrm{mL}^{-1}[4]$. The factors mentioned demand to carry out rapid, multicomponent, accurate and selective analysis for the determination of pharmaceuticals in urine.

The widely used methods for the determination of diuretics in urine are liquid and gas chromatography with mass-spectroscopic detection. These methods provide necessary selectivity and expressivity [5-13]. The main disadvantage of the methods is insufficient selectivity towards the urine components. Necessary selectivity is achieved by preliminary liquid-liquid or solid phase extraction. However, it leads to an increase in analysis time.

Other highly sensitive method of analysis of biological 
molecules is luminescent method. Isopotential fluorimetry and fluorimetry in combination with partialleast squares multivariative calibration for simultaneous determination of amiloride and triamterene in human urine are described in $[14,15]$. Although these methods are characterized by high specifity and rapidity, high dilution of urine and low recoveries of diuretics lead to low sensitivity. Besides, the interfering influence of other diuretics was not studied.

Sensitive method of determination of furosemide based on its luminescent properties was proposed by Ioannou et al. [16], but the selectivity of method was not reached. Determination of amiloride and furosemide in urine involves separation of the substances on nylon membrane [17]. The method of the luminescent determination of triamterene and its metabolite in urine demands preliminary separation on octadecyl (C18) discs that consist of glass fibber embedded with $\mathrm{C} 18$ bonded silica, providing a hydrophobic surface for retaining nonpolar compounds, and the method of determination of triamterene in pharmaceuticals - separation on MP1 (a mixed phase of nonpolar and strong cation which involves both reversed phase and cation exchange) [18]. Literature data concerning luminescent determination of other diuretics have not been found yet.

The luminescent properties of some representatives of different classes of diuretics (loop, thiazide and potassium sparing) in aqueous solutions and in human urine with the aim to develop method of determination of furosemide, bumetanide, chlorthiazide, hydrochlorothiazide, bendroflumethiazide, triamterene and amiloride were studied in present work.

\section{Materials and Methods}

\subsection{Instruments}

Luminescence spectrometer LS55 (Perkin Elmer, USA) equipped with a xenon impulse lamp source and $1.0 \mathrm{~cm}$ cell, spectrophotometer UV-VIS Unico UV-2800, pHmeter with glass electrode, centrifuge OPN-3Y4.2 (Russia), evaporator in dry nitrogen Liebisch (Germany), analytical balance KERN ABS (Germany), pipettes of adjustable volume Eppendorf (Germany) 2 - $20 \mu \mathrm{L}, 20$ - 200 $\mu \mathrm{L}, 100-1000 \mu \mathrm{L}$.

\subsection{Chemicals and Reagents}

Stock standard solutions were prepared for 7 diuretic drugs (amiloride, bendroflumethiazide, bumetanide, chlorthiazide, furosemide, hydrochlorothiazide, triamterene) (Merck, Germany) by dissolving $1.0 \mathrm{mg}$ of the diuretic in $10 \mathrm{~mL}$ of methanol (Sigma, USA, qualification "HPLC grade") to obtain concentration of $0.1 \mathrm{mg} \cdot \mathrm{mL}^{-1}$. The methanol stock solutions of diuretics were stored in a tightly closed container in a cool and dry place. Working solutions were obtained by taking an appropriate volume of standard solution, evaporating the methanol in a nitrogen stream and diluting it with water. Standard aqueous solution was freshly prepared daily.

The $\mathrm{pH}$ was regulated by adding of fixed volume of acid or alkali. Hydrochloric acid and sodium hydroxide (both Merck, Germany) were prepared by dilution of the initial concentrated solution.

Urine samples were collected from 5 volunteers who did not consume banned substances. Urine samples were stored in polypropylene bottles at a temperature of $-20^{\circ} \mathrm{C}$. Before conducting the experiment samples were defreezed and centrifuged for $10 \mathrm{~min}$ at $3000 \mathrm{rpm}$. Urine samples were diluted 10 times and the $\mathrm{pH}$ value was measured. Spiked urine samples were prepared by adding an aliquot of diuretic and appropriate volume of hydrochloric acid to negative urine samples after sample preparation to reach $\mathrm{pH} 2.0$ or 4.0 .

\section{Results and Discussion}

The diuretics selected for investigation can be classified as strong acids (bumetanide and furosemide), weak acids (thiazide derivatives) and basic compounds (triamterene and amiloride) (Table 1). Considering this fact and that

Table 1. The characteristics of selected diuretics [1].

Fendroflumethiazide


$\mathrm{pH}$ of urine of healthy person is $5.5-7.0$, it was necessary to study luminescent properties of their different protolytic forms.

It is seen that at $\mathrm{pH}$ of urine of healthy person furosemide and bumetanide exist as anions in solutions: furosemide as monoanionic and bumetanide as dianionic. The spectra of excitation and emission of molecular and anionic forms of furosemide and bumetanide are shown in Figures 1 and 2. It is seen that luminescent properties of protolytic forms of both diuretics differ appreciably. The intrinsic luminescence of furosemide at $\mathrm{pH}>5$ significantly decreases due to the destruction of rigidity of molecule which can be explained by breach of intermolecular hydrogen bonds with carboxylic groups of other furosemide molecules [17].

In contrast to furosemide the highest intensity of luminescence is peculiar to dianionic form of bumetanide,

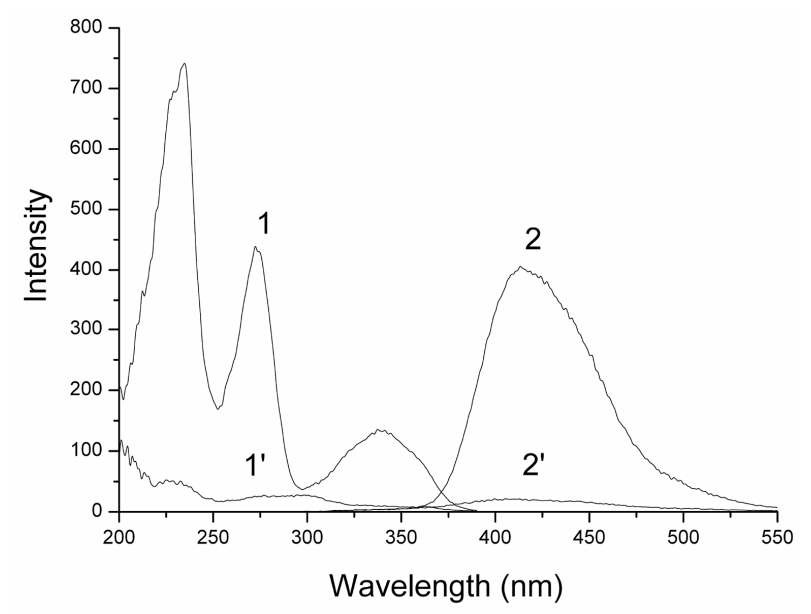

Figure 1. Excitation and emission spectra of molecular $(1,2)$ and monoanionic $\left(1^{\prime}, 2^{\prime}\right)$ forms of furosemide. $\mathrm{pH}=\mathbf{2 . 0}(1$, $2), \mathrm{pH}=6.0\left(1^{\prime}, 2^{\prime}\right), \lambda_{e x}=270 \mathrm{~nm}, \lambda_{e m}=410 \mathrm{~nm}$.

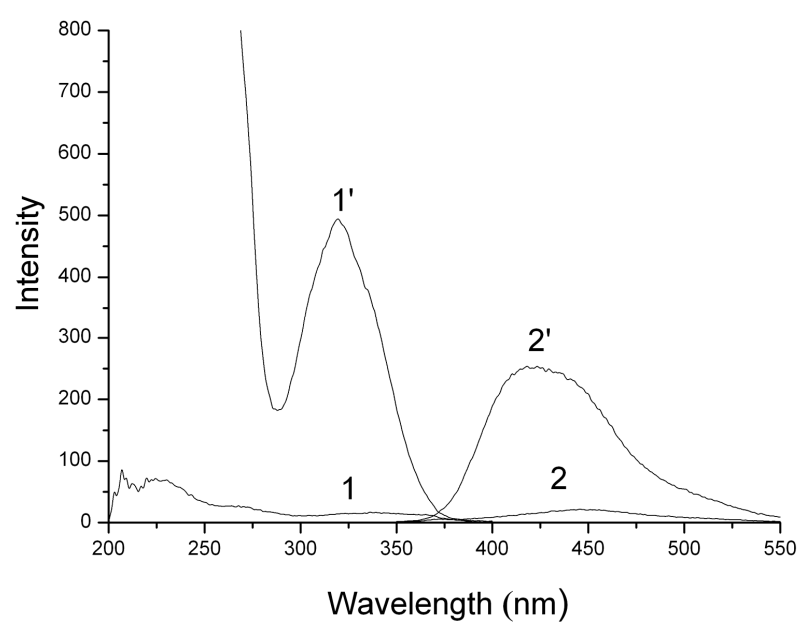

Figure 2. Excitation and emission spectra of monoanionic (1, 2) and dianionic $\left(1^{\prime}, 2^{\prime}\right)$ forms of bumetanide. $\mathrm{pH}=\mathbf{2 . 0}(1,2)$, $\mathrm{pH}=6.0\left(1^{\prime}, 2^{\prime}\right), \lambda_{e x}=325 \mathrm{~nm}, \lambda_{e m}=420 \mathrm{~nm}$. which can be explained by simultaneous deprotonation of the benzoic acid and of the anilinium ion with consequent formation of stable structure [19]. Dianionic form of bumetanide exhibits intensive excitation band at $325 \mathrm{~nm}$ with emission at $420 \mathrm{~nm}$. So, at $\mathrm{pH}$ of urine bumetanide is characterized with high luminescence intensity while furosemide demonstrates low intensity that is the evidence of possibility to determine amount of furosemide.

In acidic medium ( $\mathrm{pH}$ 2) furosemide demonstrates strong intrinsic luminescence with maxima $\lambda_{e x} / \lambda_{e m} 270 /$ $410 \mathrm{~nm}$ in contrast to bumetanide. These properties are served as the basis for the determination of furosemide in presence of commensurate amount of bumetanide after acidifying urine sample till $\mathrm{pH} 2$.

Bendroflumethiazide and hydrochlorothiazide at $\mathrm{pH}$ of urine exist in molecular form, while in case of chlorothiazide at $\mathrm{pH} 5.5$ - 7.0 molecular and monoanionic forms coexist in solution. The spectra of excitation and emission of bendroflumethiazide, hydrochlorothiazide and chlorothiazide are shown in Figures 3 and 4. The highest luminescence intensity of bendroflumethiazide is peculiar to its molecular form $\left(\lambda_{e x} / \lambda_{e m} 270 / 400 \mathrm{~nm}\right)$ which exists at $\mathrm{pH} 5.5$ - 7.0. Anionic form is characterized by negligible emission. Luminescent properties of protolytic forms of hydrochlorothiazide and chlorothiazide do not differ much. Maxima of excitation and emission for protolytic forms of both diuretics coincide which can be explained by their identical molecular structures. Luminescent intensity of chlorothiazide is 5 times more than of hydrochlorothiazide. However, considerable overlapping of excitation and emission spectra makes their simultaneous determination impossible. At the same time, determination of bendroflumethiazide in presence of commensurate amount of hydrochlorothiazide is possible due to low luminescent intensity of the latter.

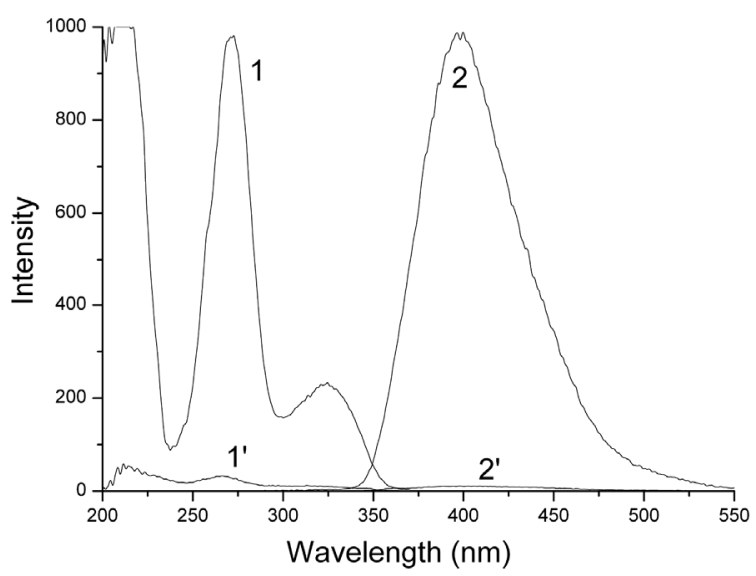

Figure 3. Excitation and emission spectra of molecular $(1,2)$ and anionic $\left(1^{\prime}, 2^{\prime}\right)$ forms of bendroflumethiazide. $\mathrm{pH}=6.0$ $(1,2), \mathrm{pH}=10.0\left(1^{\prime}, 2^{\prime}\right), \lambda_{e x}=270 \mathrm{~nm}, \lambda_{e m}=400 \mathrm{~nm}$. 
At $\mathrm{pH}$ of urine amiloride is present in solution in protonated form, and triamterene in protonated and molecular forms. Molecular forms of amiloride and triamterene are characterized with intensive luminescence (Figures 5 and 6). Protonated forms which predominate at $\mathrm{pH}$ of urine have less intensive luminescence. At $\mathrm{pH}$ 4.0 insignificant bathochromic shift for excitation $\left(\Delta \lambda_{e x}=\right.$ $6 \mathrm{~nm})$ and emission maxima $\left(\Delta \lambda_{e m}=4 \mathrm{~nm}\right)$ is observed. This fact can be explained by protonation of aminogroupes [18].

Determination of amiloride in mixture with triamterene is impossible due to high luminescent intensity of the latter. However, triamterene can be determined in presence of any amount of amiloride. Elaborated method of determination of triamterene as individual substance in aqueous solution without pre-concentration demonstrates

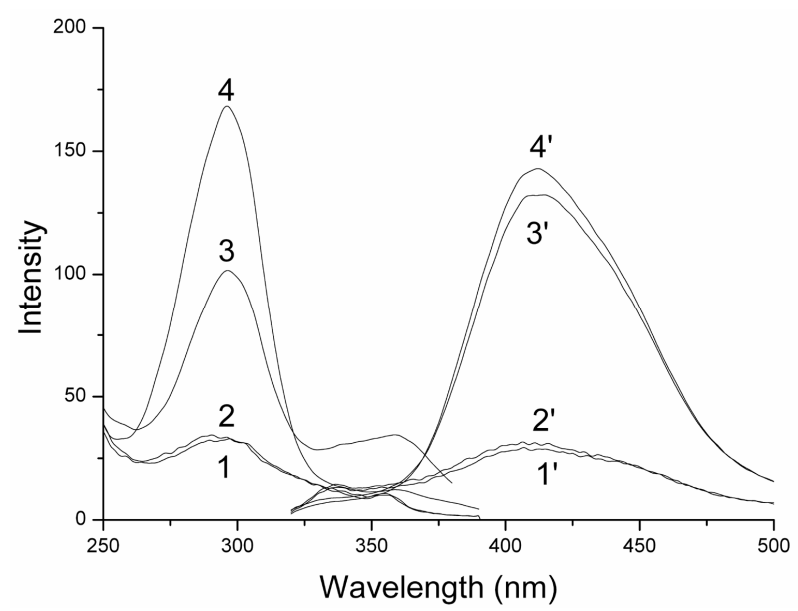

Figure 4. Excitation and emission spectra of molecular $\left(1,1^{\prime}\right)$ and anionic $\left(2,2^{\prime}\right)$ forms of hydrochlorothiazide and of molecular $\left(3,3^{\prime}\right)$ and anionic $\left(4,4^{\prime}\right)$ forms of chlorothiazide. $\mathrm{pH}$ $=6.0(1,3), \mathrm{pH}=10.0(2,4), \lambda_{e x}=295 \mathrm{~nm}, \lambda_{e m}=405 \mathrm{~nm}$.

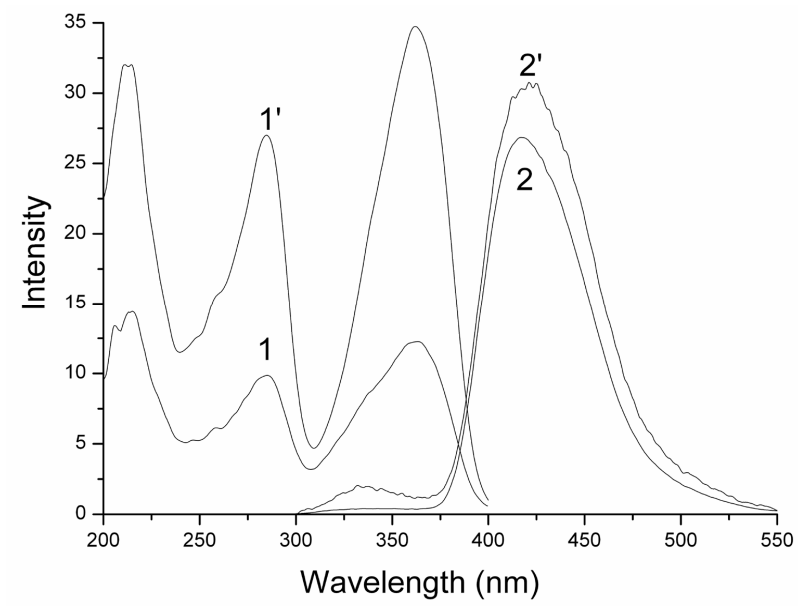

Figure 5. Excitation and emission spectra of protonated (1, 2) and molecular $\left(1^{\prime}, 2^{\prime}\right)$ forms of amiloride. $\mathrm{pH}=6.0(1,2)$, $\mathrm{pH}=10.0\left(1^{\prime}, 2^{\prime}\right), \lambda_{e x}=285 \mathrm{~nm}, \lambda_{e m}=420 \mathrm{~nm}$.

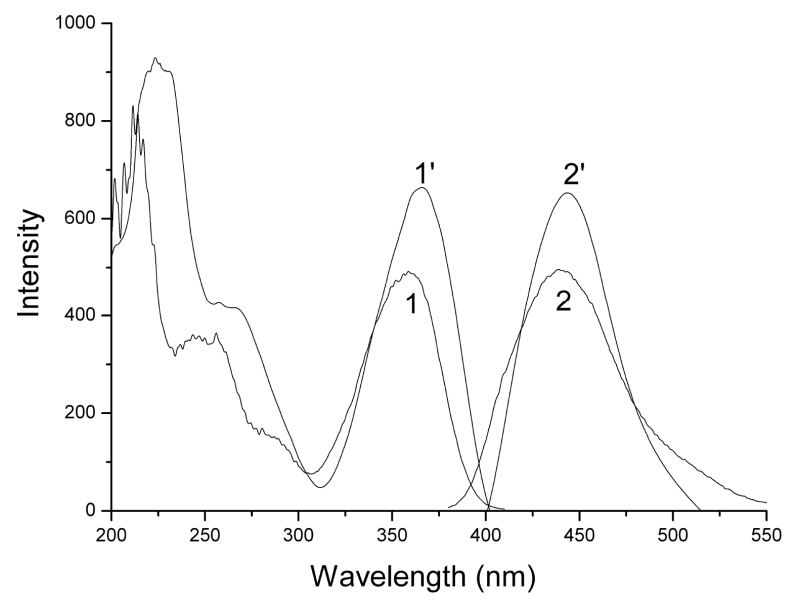

Figure 6. Excitation and emission spectra of protonated (1, 2) and molecular $\left(1^{\prime}, 2^{\prime}\right)$ forms of triamterene. $\mathrm{pH}=\mathbf{6 . 0}(1$, $2), \mathrm{pH}=10.0\left(1^{\prime}, 2^{\prime}\right), \lambda_{e x}=360 \mathrm{~nm}, \lambda_{e m}=440 \mathrm{~nm}$.

the results competitive with that obtained in [18]. The same LOD and linearity range were achieved without solid-phase extraction which decreased time of analysis and simplified the method of determination.

The excitation and emission intensities as function of diuretic concentration under optimal conditions were studied. Limit of Detection was calculated as relation of threefold standard deviation of noise signal to coefficient of instrumental sensitivity. The results are shown in Table 2.

\subsection{Interfering Influence}

The highest sensitivity is inherent for the methods of determination of furosemide and triamterene as it can be seen in Table 2. At $\mathrm{pH}$ of urine all diuretics except furosemide show luminescent properties. Furosemide at $\mathrm{pH}>5$ does not interfere with determination of other diuretics but all diuretics except bumetanide interfere with its determination. Furosemide and bumetanide can be determined in one sample by adjusting $\mathrm{pH}$. So, at $\mathrm{pH}$ 2.0 bumetanide molecule does not emit light which allows determination of furosemide. On the contrary, at $\mathrm{pH} 6.0$ bumetanide is characterized with high luminescence intensity, and furosemide molecule does not emit light. The possibility of determination of one diuretic in presence of other in commensurate amount with sufficient sensitivity was proved. Results are shown in Table 3.

Due to overlapping of spectra of all diuretics and to high emission intensity triamterene at optimal conditions and at $\mathrm{pH}$ of urine impedes determination of studied diuretics, but diuretics in concentration lower or equal to concentration of triamterene do not impede its determination. Thus, for determination of other diuretics in presence of triamterene its preliminary separation is indispensable. 
Table 2. Some analytical figures of merit of determination of diuretics by intrinsic luminescence in aqueous solutions. $R^{2}=$ 0.998 - 0.999 .

\begin{tabular}{|c|c|c|c|c|c|c|c|}
\hline \multirow{2}{*}{ Diuretic } & \multirow{2}{*}{$\mathrm{pH}$} & \multirow{2}{*}{$\lambda_{\mathrm{em}}^{\max }\left(\lambda_{\mathrm{ex}}^{\max }\right), \mathrm{nm}$} & \multicolumn{3}{|c|}{$I=(\mathrm{a} \pm \Delta a)+(b \pm \Delta b) \mathrm{C}, \mu \mathrm{g} \cdot \mathrm{mL}^{-1}$} & \multirow{2}{*}{$\begin{array}{l}\mathrm{LOD}, \\
\mu \mathrm{g} \cdot \mathrm{L}^{-1}\end{array}$} & \multirow{2}{*}{$\begin{array}{c}\text { Linearity range } \\
\mu \mathrm{g} \cdot \mathrm{L}^{-1}\end{array}$} \\
\hline & & & $I$ & $a \pm \Delta a$ & $b \pm \Delta b$ & & \\
\hline \multirow{3}{*}{ Furosemide } & \multirow{3}{*}{2.0} & \multirow{3}{*}{$410(270)$} & $I_{e x}$ & $9 \pm 4$ & $1988 \pm 19$ & 6.0 & \multirow{3}{*}{$10-2000$} \\
\hline & & & & & & & \\
\hline & & & $I_{e m}$ & $14 \pm 6$ & $1980 \pm 26$ & 9.0 & \\
\hline \multirow{3}{*}{ Bumetanide } & \multirow{3}{*}{6.0} & \multirow{3}{*}{$420(325)$} & $I_{e x}$ & $3 \pm 4$ & $392 \pm 4$ & 30 & \multirow{3}{*}{$50-5000$} \\
\hline & & & & & & & \\
\hline & & & $I_{e m}$ & $1 \pm 2$ & $395 \pm 2$ & 20 & \\
\hline \multirow{3}{*}{ Bendroflumethiazide } & \multirow{3}{*}{6.0} & \multirow{3}{*}{$400(270)$} & $I_{e x}$ & $16 \pm 5$ & $988 \pm 13$ & 20 & \multirow{3}{*}{$10-5000$} \\
\hline & & & & & & & \\
\hline & & & $I_{e m}$ & $17 \pm 6$ & $992 \pm 15$ & 20 & \\
\hline \multirow{3}{*}{ Amiloride } & \multirow{3}{*}{6.0} & \multirow{3}{*}{$420(285)$} & $I_{e x}$ & $12 \pm 7$ & $489 \pm 7$ & 40 & \multirow{3}{*}{$50-5000$} \\
\hline & & & $I$ & $16+6$ & $413+6$ & 40 & \\
\hline & & & & & & & \\
\hline \multirow{3}{*}{ Triamterene } & \multirow{3}{*}{4.0} & \multirow{3}{*}{$440(360)$} & $I_{e x}$ & $1 \pm 1$ & $3805 \pm 26$ & 0.8 & \multirow{3}{*}{$1-1000$} \\
\hline & & & & & & & \\
\hline & & & $I_{e m}$ & $2 \pm 1$ & $3802 \pm 3$ & 0.8 & \\
\hline
\end{tabular}

Table 3. Determination of furosemide (Fur) and bumetanide (Bum) in aqueous solutions.

\begin{tabular}{|c|c|c|c|c|c|c|c|c|}
\hline \multirow{2}{*}{$\mathrm{pH}$} & \multicolumn{2}{|c|}{ Added, $\mu \mathrm{g} \cdot \mathrm{L}^{-1}$} & \multicolumn{2}{|c|}{ Found, $\mu \mathrm{g} \cdot \mathrm{L}^{-1}$} & \multicolumn{2}{|c|}{ Recovery, \% } & \multicolumn{2}{|c|}{ RSD, \% } \\
\hline & Fur & Bum & Fur & Bum & Fur & Bum & Fur & Bum \\
\hline \multirow{3}{*}{2.0} & 0 & 200 & 0 & 2.8 & 0 & 1.4 & - & 8.8 \\
\hline & 200 & 0 & 198.6 & 0 & 99.3 & 0 & 3.7 & - \\
\hline & 200 & 200 & 203 & 0 & 101.5 & 0 & 6.9 & - \\
\hline \multirow{3}{*}{6.0} & 0 & 200 & 0 & 197 & 0 & 98.5 & - & 4.0 \\
\hline & 200 & 0 & 5.1 & 0 & 2.6 & 0 & 8.9 & - \\
\hline & 200 & 200 & 0 & 205 & 0 & 102.5 & - & 3.8 \\
\hline
\end{tabular}

\subsection{Urine Analysis}

Determination of diuretics in urine is complicated due to the influence of matrix which contains a variety of organic substances. Most of these substances exhibit high absorbance in the ultraviolet region [20] and have strong background luminescence that interferes with the direct determination of diuretics. As a result, the urine must be diluted and the fluorescence intensity should be measured at the maximum of the highest excitation wavelength where the urine exhibits low absorbance.

Dilution in 10 times and measuring of intensity signal versus blank urine was proved to be effective in order to avoid an interfering influence of matrix and distortion of the spectra. As it was established earlier the highest luminescence intensity was inherent to molecular forms of triamterene and amiloride $(\mathrm{pH}>8.0$ and 9.5, respectively), but alkalization of urine till $\mathrm{pH} 9.5-10.0$ leads to appearance of turbidity. Thus, to avoid additional step in sample preparation including filtration determination of these diuretics was realized at $\mathrm{pH} 4.0$ at which both diuretics exist in protonated forms.

As it can be seen from Figure 7 triamterene in urine is characterized by high intensive excitation and emission with maxima at 370 and $440 \mathrm{~nm}$, respectively.

Triamterene as individual substance can be determined in urine by either excitation or emission intensity with LOD obtained 5 and $6 \mu \mathrm{g} \cdot \mathrm{L}^{-1}$, respectively. Considering the dilution of urine in 10 times, LOD is higher than that in aqueous solutions but is still enough to detect triamterene with necessary sensitivity. The linearity range is 
$20-500 \mu \mathrm{g} \cdot \mathrm{L}^{-1}$.

Amiloride does not exhibit luminescence in urine (Figure 8) which can be explained by binding with urine components such as derivatives of kynurenine, xanthurenic and folic acids [20]. As individual substance it can be determined by excitation spectra with LOD $30 \mu \mathrm{g} \cdot \mathrm{L}^{-1}$ and linearity range $200-2000 \mu \mathrm{g} \cdot \mathrm{L}^{-1}$. The presence of triamterene makes determination of amiloride by excitation spectra impossible since their maxima coincide. Overlapping leads to the increase of total signal intensity. However, determination of amiloride in presence of any amount of triamterene is still manageable by spectrophotometry, by intrinsic absorbance at $360 \mathrm{~nm}$ (Figure 9), in area where other diuretics in urine do not absorb. The linearity range is $50-1000 \mu \mathrm{g} \cdot \mathrm{L}^{-1}$ with LOD 10 $\mu \mathrm{g} \cdot \mathrm{L}^{-1}$ obtained. Determination of triamterene in presence of any amount of amiloride is possible by its emission spectrum at wavelength $440 \mathrm{~nm}$.

High recoveries of amiloride and triamterene from

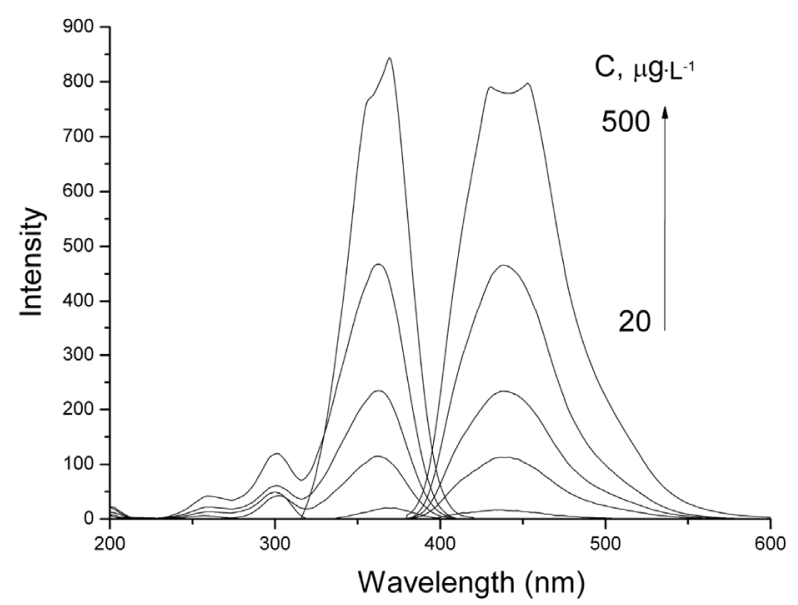

Figure 7. Excitation and emission spectra of triamterene in urine. $\mathrm{C}=20-500 \mu \mathrm{g} \cdot \mathrm{L}^{-1} \cdot \mathrm{pH}=4.0 . \lambda_{e x}=360 \mathrm{~nm}, \lambda_{e m}=440$ nm.

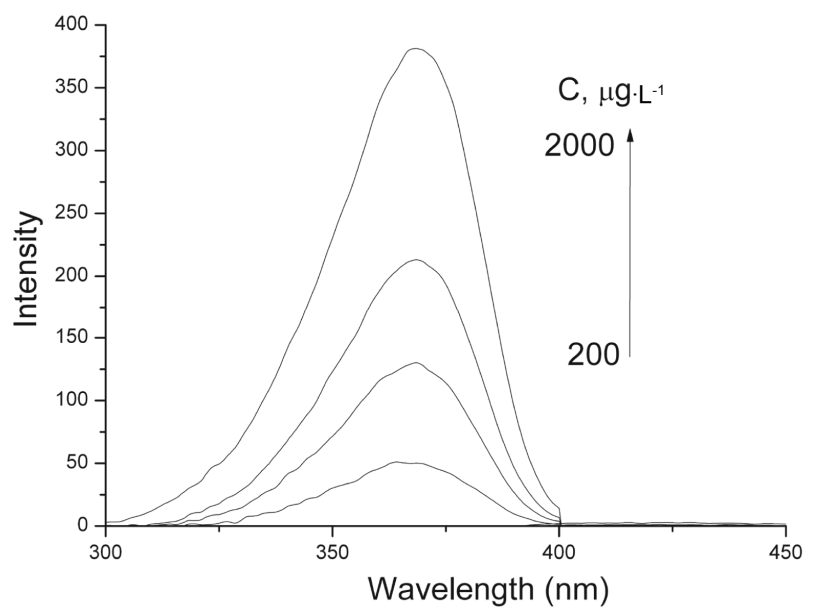

Figure 8. Excitation spectrum of amiloride in urine $\mathbf{C}=\mathbf{2 0 0}$ $-2000 \mu \mathrm{g} \cdot \mathrm{L}^{-1} \cdot \mathrm{pH}=4.0 . \lambda_{e x}=285 \mathrm{~nm}, \lambda_{e m}=420 \mathrm{~nm}$.

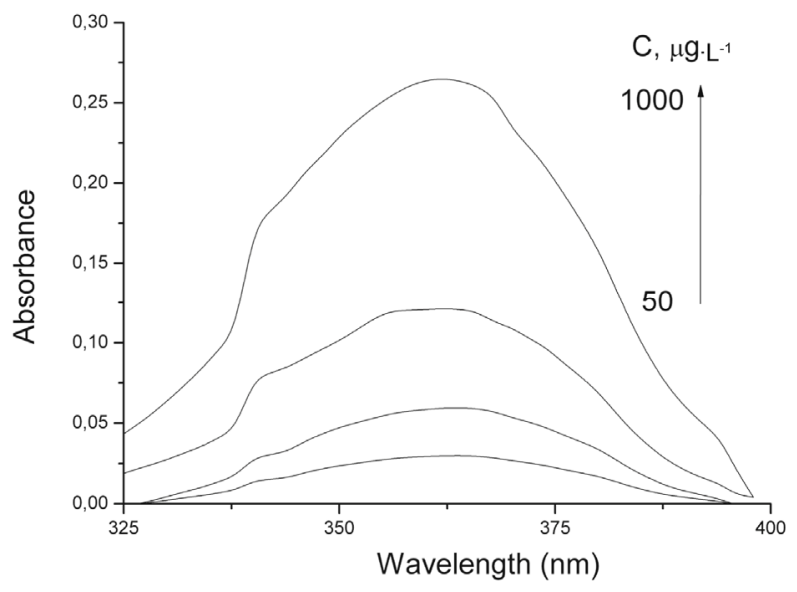

Figure 9. Spectrum of absorbance of amiloride in urine. $\mathrm{C}=$ $50-1000 \mu \mathrm{g} \cdot \mathrm{L}^{-1} \cdot \mathrm{pH}=4.0 . \lambda=360 \mathrm{~nm}$.

human urine and low relative standard deviations (Table 4) are the evidence of accuracy and reproducibility of the method given. Retaining of necessary specifity on reduction the analysis time compared with that proposed in [18] owing to avoiding solid-phase extraction is important achievement of our work.

Bumetanide, furosemide and bendroflumethiazide were proved to exhibit high luminescence under optimal conditions in aqueous solutions. The influence of complex urine matrix leads to disappearance of luminescent properties of these diuretics at optimal conditions. Luminescence quenching probably can be explained by their binding with components of urine, supposedly with aminoacids [20]. So, determination of bumetanide, furosemide and bendroflumethiazide assume preliminary sample preparation of urine.

\section{Conclusions}

Intrinsic luminescent properties of different protolytic forms of furosemide, bumetanide, bendroflumethiazide, amiloride and triamterene are taken as a principle of sensitive determination of the diuretics in aqueous solutions. The simplicity and rapidity of method make it a useful tool in determination.

Techniques elaborated for determination of triamterene in presence of other diuretics and furosemide in presence of commensurate amount of bumetanide allow enhancing specifity of analysis. Other studied diuretics can not be determined in presence of triamterene which requires its preliminary separation.

Dilution of urine and measuring the signal versus blank solution allowed avoiding matrix influence in case of triamterene and amiloride analysis and elaborating simple, selective and sensitive method for their determination. Other diuretics suppose to be binded with components of urine resulting in luminescence quenching 
Table 4. Analytical recoveries of amiloride and triamterene from human urine.

\begin{tabular}{|c|c|c|c|c|c|c|c|c|c|c|}
\hline \multicolumn{7}{|c|}{ Amiloride } & \multicolumn{4}{|c|}{ Triamterene } \\
\hline $\begin{array}{l}\text { Added, } \\
\mu \mathrm{g} \cdot \mathrm{L}^{-1}\end{array}$ & Found $^{* a}, \mu \mathrm{g} \cdot \mathrm{L}^{-1}$ & $\begin{array}{c}\text { Recovery, } \\
\%\end{array}$ & $\begin{array}{c}\text { RSD, } \\
\%\end{array}$ & $\begin{array}{c}\text { Found }{ }^{* \mathrm{~b}} \\
\mu \mathrm{g} \cdot \mathrm{L}^{-1}\end{array}$ & $\begin{array}{c}\text { Recovery, } \\
\%\end{array}$ & $\begin{array}{l}\text { RSD, } \\
\%\end{array}$ & $\begin{array}{l}\text { Added, } \\
\mu \mathrm{g} \cdot \mathrm{L}^{-1}\end{array}$ & $\begin{array}{l}\text { Found", } \\
\mu \mathrm{g} \cdot \mathrm{L}^{-1},\end{array}$ & $\begin{array}{c}\text { Recovery, } \\
\%\end{array}$ & $\mathrm{RSD}, \%$ \\
\hline 225 & 227 & 100.9 & 8.7 & 228 & 101.3 & 5.7 & 22.5 & 22.7 & 100.9 & 6.2 \\
\hline 400 & 397 & 99.2 & 6.2 & 402 & 100.5 & 6.4 & 90 & 88.6 & 98.4 & 5.9 \\
\hline 1000 & 998 & 99.8 & 6.4 & 1000 & 100.0 & 6.5 & 250 & 252 & 100.8 & 5.8 \\
\hline 1500 & 1508 & 100.5 & 5.3 & 1510 & 100.6 & 5.5 & 400 & 396 & 99.0 & 7.6 \\
\hline
\end{tabular}

*Average of 3 measurements. ${ }^{\text {a }}$ Quantity found by luminescent method. ${ }^{b}$ Quantity found by spectrophometry.

which demands preliminary sample preparation of urine.

Triamterene in urine can be determined in presence of studied diuretics by emission intensity with high sensitivity. Simple method of amiloride determination in human urine was proposed. It does not include long sample preparation but provides high specifity of analysis with sufficient sensitivity. The reduction of time of analysis due to avoiding sample preparation merits the techniques proposed.

\section{REFERENCES}

[1] R. Ventura and J. Segura, "Detection of Diuretic Agents in Doping Control," Journal of Chromatography B: Biomedical Sciences and Applications, Vol. 687, No. 1, 1996, pp. 127-144.

http://dx.doi.org/10.1016/S0378-4347(96)00279-4

[2] A. Morganti, "Should a Diuretic Always Be the First Choice in Patients with Essential Hypertension? The Case for No," Journal of the American Society of Nephrology, Vol. 16, No. 3, 2005, pp. 70-73. http://dx.doi.org/10.1681/ASN.2004110964

[3] WADA, "The World Anti-Doping Code-The 2009 Prohibited List: International Standard," World Anti-Doping Agency, Montreal, 2009.

[4] WADA, "Minimum Required Performance Limits for Detection of Prohibited Substances (Technical Document TD2009MRPL)," World Anti-Doping Agency, Montreal, 2009.

[5] C. Brunelli, C. Bicchi, A. Di Stilo, A. Salomone and M. Vincenti, "High-Speed Gas Chromatography in Doping Control: Fast-GC and Fast-GC/MS Determination of $\beta$ Adrenoceptor Ligands and Diuretics," Journal of Separation Science, Vol. 29, No. 18, 2006, pp. 2765-2771. http://dx.doi.org/10.1002/jssc.200500387

[6] R. Ventura, M. Roig, N. Monfort, P. Saez, R. Berges and J. Segura, "High-Throughput and Sensitive Screening by Ultra-Performance Liquid Chromatography Tandem Mass Spectrometry of Diuretics and Other Doping Agents," European Journal of Mass Spectrometry, Vol. 14, No. 3, 2008, pp. 191-200. http://dx.doi.org/10.1255/ejms.920

[7] M. Mazzarino, X. de la Torre and F. Botrè, "A Screening Method for the Simultaneous Detection of Glucocorticoids, Diuretics, Stimulants, Anti-Estrogens, Beta-Adre- nergic Drugs and Anabolic Steroids in Human Urine by LC-ESI-MS/MS," Analytical and Bioanalytical Chemistry, Vol. 392, No. 4, 2008, pp. 681-698.

http://dx.doi.org/10.1007/s00216-008-2292-5

[8] O. J. Pozo, P. Van Eenoo, K. Deventer and F. T. Delbeke, "Development and Validation of a Qualitative Screening Method for the Detection of Exogenous Anabolic Steroids in Urine by Liquid Chromatography-Tandem Mass Spectrometry," Analytical and Bioanalytical Chemistry, Vol. 389, No. 4, 2007, pp. 1209-1224.

http://dx.doi.org/10.1007/s00216-007-1530-6

[9] K. Deventer, O. J. Pozo, P. Van Eenoo and F. T. Delbeke, "Qualitative Detection of Diuretics and Acidic Metabolites of Other Doping Agents in Human Urine by High-Performance Liquid Chromatography-Tandem Mass Spectrometry. Comparison between Liquid-Liquid Extraction and Direct Injection," Journal of Chromatography A, Vol. 1216, No. 31, 2009, pp. 5819-5827. http://dx.doi.org/10.1016/j.chroma.2009.06.003

[10] V. Morra, P. Davit and P. Capra, "Fast Gas Chromatographic/Mass Spectrometric Determination of Diuretics and Masking Agents in Human Urine. Development and Validation of a Productive Screening Protocol for Antidoping Analysis," Journal of Chromatography A, Vol. 1135, No. 2, 2006, pp. 219-229.

http://dx.doi.org/10.1016/j.chroma.2006.09.034

[11] L. Amendola, C. Colamonici, M. Mazzarino and F. Botrè, "Rapid Determination of Diuretics in Human Urine by Gas Chromatography-Mass Spectrometry Following Microwave Assisted Derivatization," Analytica Chimica Acta, Vol. 475, No. 1-2, 2003, pp. 125-136. http://dx.doi.org/10.1016/S0003-2670(02)01223-0

[12] Yi.-L. Tseng, M.-H. Shieh, Ch.-Ts. Lin and F.-H. Kuo, "Detection of Diuretics in Urine during Sports Events in Taiwan," Tzu Chi Medical Journal, Vol. 16, No. 2, 2004, pp. 69-77.

[13] O. Zaporozhets, I. Tsyrulneva and M. Ischenko, "Determination of 8 Diuretics and Probenecid in Human Urine by Gas Chromatography-Mass Spectrometry: Confirmation Procedure," American Journal of Analytical Chemistry, Vol. 3, No. 4, 2012, pp. 320-327. http://dx.doi.org/10.4236/ajac.2012.34044

[14] J. A. M. Pulgarin, A. A. Molina and P. F. Lopez, "Direct Analysis of Amiloride and Triamterene Mixtures by Fluorescence Spectrometry Using Partial-Least Squares Calibration," Analytica Chimica Acta, Vol. 449, No. 1-2, 
2001, pp. $179-187$.

http://dx.doi.org/10.1016/S0003-2670(01)01356-3

[15] J. A .M. Pulgarin, A. A. Molina and P. F. Lopez, "Simultaneous Direct Determination of Amiloride and Triamterene in Urine Using Isopotential Fluorometry," Analytical Biochemistry, Vol. 292, No. 1, 2001, pp. 59-68. http://dx.doi.org/10.1006/abio.2001.5064

[16] P. C. Ioannou, N. V. Rusakova, D. A. Andrikopoulou, K. M. Glynou and G. M. Tzompanakia, "Spectrofluorimetric Determination of Anthranilic Acid Derivatives Based on Terbium Sensitized Fluorescence," Analyst, Vol. 123, No. 12, 1998, pp. 2839-2843. http://dx.doi.org/10.1039/a806093b

[17] C. M. Peralta, L. P. Fernández and A. N. Masi, "Solid Phase Extraction Using Nylon Membranes with Fluorescence Detection as a Fast and Sensitive Method for Amiloride and Furosemide Determination in Urine Samples," Microchemical Journal, Vol. 98, No. 1, 2011, pp.
39-43. http://dx.doi.org/10.1016/j.microc.2010.10.009

[18] G. A. Ibanez, G. M. Escandar, A. E. Mansilla and A. M. de la Pena, "Determination of Triamterene in Pharmaceutical Formulations and of Triamterene and Its Main Metabolite Hydroxytriamterene Sulfate in Urine Using SolidPhase and Aqueous Solution Luminescence," Analytica Chimica Acta, Vol. 538, No. 1-2, 2005, pp. 77-84. http://dx.doi.org/10.1016/j.aca.2005.02.001

[19] B. Song, A. K. Galande, K. Kodukula, W. H. Moos and S. M. Miller, "Evaluation of the $\mathrm{PK}_{\mathrm{a}}$ Values and Ionization Sequence of Bumetanide Using ${ }^{1} \mathrm{H}$ and ${ }^{13} \mathrm{C}$ NMR and UV Spectroscopy," Drug Development Research, Vol. 72, No. 5, 2011, pp. 416-426. http://dx.doi.org/10.1002/ddr.20443

[20] M. J. P. Leiner, M. R. Hubmann and O. S. Wolfbeis, "The Total Fluorescence of Human Urine," Analytica Chimica Acta, Vol. 198, 1987, pp. 13-23. http://dx.doi.org/10.1016/S0003-2670(00)85002-3 